\title{
A DERIVATION OF DEGENERATE VON KÁRMÁN EQUATIONS FOR STRONGLY ANISOTROPIC PLATES
}

\author{
$\mathrm{BY}$ \\ ROBERT G. ROOT \\ Department of Mathematics, Lafayette College, Easton, Pennsylvania
}

1. Introduction. This paper derives a boundary value problem for a nonlinear, degenerate elliptic system of partial differential equations modeling the deformation of an anisotropic plate. The principal result is that stress and displacement for the threedimensional object can be approximately reconstructed from the solutions of a twodimensional problem that approximates the three-dimensional problem with error of the order of the thickness of the plate. The derivation is based on that for the von Kármán plate model by Ciarlet in [2]. The classical von Kármán equations are a bidimensional model for the deflection of isotropic plates dating back to von Kármán's work [15] in 1910. Ciarlet's derivation for the isotropic case is the most convincing justification to date for the validity of these equations based on the equations of three-dimensional elasticity. The derivation hinges on the assumption that the Young's modulus of the plate is large with respect to the thickness. Young's modulus is a constitutive constant measuring the stiffness of the plate. For isotropic plates, and plates with only "mild" anisotropy (with slightly different stiffnesses in different directions), the assumption that the Young's modulus is adequately large is not an imposition. If the Young's modulus is unacceptably small, then the "plate" should be thought of as a membrane. On the other hand, consider the case of a "strongly" anisotropic plate, that is, a plate whose stiffness in one direction is very small in comparison with that in the perpendicular direction. A common example is a sheet of corrugated cardboard, which bends much more easily with the corrugations than across them. In this case it may be that the stiffness in one direction is of sufficient magnitude to demand a plate model, while that in the other is more appropriate to a membrane model. This is the situation treated here.

The resulting model includes a fourth-order partial differential operator with positive semi-definite characteristic form. This badly degenerate form of ellipticity we call here elliptic-parabolic, after Fichera's second-order equations, presented in [5], and widely known. Such operators have appeared in linear models for plates that lose stiffness in Weinacht's work [17], and the author's [12]. Greenlee in [7] has examined the related case of a plate/membrane system. To the author's knowledge, this paper is the first derivation

Received July 12, 1994.

1991 Mathematics Subject Classification. Primary 73C30; Secondary 35J70, 35G05.

(C)1999 Brown University 
of a nonlinear model of this form. The companion article, Boundary value problems for degenerate von Kármán equations, by the author, presents regularity results that permit a weak formulation of the problem amenable to the application of monotone operator theory like that presented in [1].

In our derivation, we begin with the equations of three-dimensional elastic equilibrium. Readers unfamiliar with these equations might read an introduction to finite elasticity. Stoker's book [14] has attained the stature and longevity of a classic. Marsden and Hughes provide a superb introduction in [9], although with a more geometric flavor than the work at hand. More succinct is Gurtin's monograph [6]. Finally, one should not overlook Ciarlet and Rabier's French exposition in [4]. The first two sections of the first chapter are an admirable introduction to this paper, since the work here is inspired by the derivation that completes the chapter. The work done there is generalized and extended in the important monograph [3].

2. Notation. The region $\Omega^{\epsilon} \in \mathbf{R}^{3}$ is the volume occupied by the plate in its reference state. It can be decomposed as $\Omega^{\epsilon}:=\omega \times(-\epsilon, \epsilon)$ where $\omega \in \mathbf{R}^{2}$ is the outline of the plate: a bounded, open, simply connected set that we assume has a piecewise smooth boundary, and $2 \epsilon$ is the uniform thickness of the plate (assumed to be small). We denote the boundary of $\omega$ by $\partial \omega=: \gamma$, and decompose the boundary of $\Omega^{\epsilon}$ by:

$$
\begin{array}{lrl}
\Gamma_{+}^{\epsilon}:=\bar{\omega} \times\{\epsilon\}, & \Gamma_{ \pm}^{\epsilon}:=\Gamma_{+}^{\epsilon} \cup \Gamma_{-}^{\epsilon}, \\
\Gamma_{0}^{\epsilon}:=\gamma \times(-\epsilon, \epsilon), & \Gamma_{0}^{\epsilon} \cup \Gamma_{+}^{\epsilon} \cup \Gamma_{-}^{\epsilon}=\partial \Omega^{\epsilon} . \\
\Gamma_{-}^{\epsilon}:=\bar{\omega} \times\{-\epsilon\}, &
\end{array}
$$

On the boundary of any region (two- or three-dimensional) we denote the unit outer normal by $\mathbf{n}$ with individual components $n_{i}$. The corresponding directional derivative is $\frac{\partial}{\partial \mathbf{n}}$. The tangential derivative in the counterclockwise direction around $\gamma$ is $\frac{d}{d s}:=$ $-n_{2} \frac{\partial}{\partial x_{1}}+n_{1} \frac{\partial}{\partial x_{2}}$.

The three-dimensional vector field $\mathbf{u}\left(x_{1}, x_{2}, x_{3}\right):=\left(u_{1}, u_{2}, u_{3}\right)$ is the displacement function describing the deformed plate. The component $u_{i}\left(x_{1}, x_{2}, x_{3}\right)$ is the displacement of the point $\left(x_{1}, x_{2}, x_{3}\right)$ in the $x_{i}$ direction in the deformed state. The domain of $\mathbf{u}$ is $\Omega^{\epsilon}$. We use tensor notation for differentiation: $u_{i, j}:=\frac{\partial u_{i}}{\partial x_{j}}$.

The Cauchy stress tensor associated with the deformation $\mathbf{u}$ is denoted by $\sigma$. We represent it in the given coordinates by the symmetric three by three matrix with entries $\sigma_{i j}$. The symbol $\varepsilon$ denotes the strain; the exact definition is given below in the constitutive assumptions in Sec. 4. Note that $\epsilon$ and $\varepsilon$ are not equivalent in our notation.

We indicate the range of an index by the alphabet from which it comes. Greek letters range over 1 and 2, while Latin letters range over 1, 2, and 3. The convention is particularly useful when we employ the summation convention; terms with a repeated index should be summed over the range of that index. We assume this convention below, except where specifically noted. When working with Greek indices we will find it convenient to adopt the notation $\sim \alpha$ for the index not represented by $\alpha$, e.g., if $\alpha=1$, then $\sim \alpha=2$. 
3. Statement of the model, and outline of the derivation. The model presented here is based on the classical von Kármán equations for an isotropic plate; they are

$$
\begin{gathered}
\frac{2 E \epsilon^{3}}{3\left(1-\nu^{2}\right)} \Delta^{2} u-[u, w]=f \quad \text { in } \omega \\
\Delta^{2} w+\frac{E}{2}[u, u]=0 \quad \text { in } \omega .
\end{gathered}
$$

The bilinear form is defined by

$$
[u, w]:=(-1)^{\alpha+\beta} u_{, \alpha \beta} w_{, \sim \alpha \sim \beta},
$$

while $\Delta^{2}$ represents the biharmonic operator

$$
\Delta^{2} u=u_{, 1111}+u_{, 2222}+2 u_{, 1212} .
$$

The boundary conditions, assuming a clamped plate, are

$$
u=\frac{\partial u}{\partial \mathbf{n}}=0 \quad \text { and } \quad-\frac{d}{d s} w_{, 1}=h_{2}, \quad \frac{d}{d s} w_{, 2}=h_{1} \quad \text { on } \partial \omega .
$$

Here, $u$ is the vertical deflection of the centerplane of the plate under the load $f$ with the forces $h_{1}$ and $h_{2}$ being applied in the $x_{1}$ and $x_{2}$ directions respectively, along the edge of the plate. The function $w$ is the accompanying Airy stress function, from which the in-plane (meaning in the centerplane) stress tensor may be deduced. The constants $E$ and $\nu$ are the Young's modulus and Poisson's ratio (another constitutive constant; see the constitutive assumptions in the next section), respectively, and $\epsilon$ is half the thickness of the plate.

In this paper we assume that the Young's modulus in the $x_{1}$ direction is less than that in the $x_{2}$ direction by a factor $\epsilon$, half the plate's thickness; we refer to this as an order of magnitude. We derive a two-dimensional problem similar to the von Kármán problem above. The model constructed here is:

$$
\begin{gathered}
\frac{\epsilon^{2}}{3}\left(E u_{, 2222}+4 G u_{, 1212}\right)-[u, w]=f \text { in } \omega \\
\frac{1}{E}\left(w_{, 1111}+w_{, 2222}\right)+\frac{1-\frac{\nu}{E}}{G} w_{, 1212}=-\left(u_{, 1} u_{, 22}\right)_{, 1} \quad \text { in } \omega
\end{gathered}
$$

under the boundary conditions:

$$
\begin{aligned}
u & =0 & & \text { on } \partial \omega, \\
\frac{\partial u}{\partial \mathbf{n}} & =0 & & \text { on } \partial \omega \text { where } n_{2} \neq 0, \\
-\frac{d}{d s} w_{, 1} & =h_{2}, & & \text { and } \frac{d}{d s} w_{, 2}=h_{1} \quad \text { on } \partial \omega .
\end{aligned}
$$

Here we use the same notation as in the von Kármán equations above, but $E$ is the Young's modulus in the $x_{2}$ direction, and $G$ is the in-plane shear modulus. The first equation above is an elliptic-parabolic fourth-order equation in the deflection ( $u$ here, referred to as $u_{3}$ in the derivation) with second-order coefficients given by the Airy stress function. The second equation equates a uniformly elliptic fourth-order operator of the stress function to a degenerate quadratic form acting on $u$. This form omits exactly 
those derivatives over which the elliptic-parabolic equation in the deflection does not give us control. This lends credence to the possibility of solving the derived problem under appropriate conditions; a weak formulation is given in [13].

The three-dimensional problem is carefully described in Sec. 4. The derivation of the two-dimensional problem consists of three steps. We begin in Sec. 5 with a change of variables that maps the plate onto a body with the same outline but with thickness two. In making this change of variables we place weights on the solutions of the problem in the new domain. These weights carry implicit assumptions about the relative sizes of the various components of the solutions. Because of the anisotropy, we cannot simply apply the same weights Ciarlet used for the isotropic von Kármán case.

There are two changes of weights that seem fruitful. One is to assume that the tensile stress in the $x_{1}$ direction, $\sigma_{11}$, is larger than the other in-plane components of the stress tensor by a factor of the square root of the thickness. It is possible to derive a two-dimensional model with this assumption, but it leaves us with an untenable difficulty. Either we are faced with a doubly degenerate system of equations, for which it is doubtful that a suitable existence theorem can be found, or we must perform a change of variables elongating the plate's outline by a factor of $\epsilon$. Since the model is founded on the assumption that $\epsilon$ grows small asymptotically, the change of variables is undesireable. Instead, we assume that the displacement in the $x_{1}$ direction, $u_{1}$, is an order of magnitude larger than in the $x_{2}$ direction, $u_{2}$. Physically, this is reasonable, since a strongly anisotropic plate undergoes more stretching in the $x_{1}$ direction than an isotropic plate under the same load, in order to generate sufficient tension to support the load. At first this seems fruitless, since the von Kármán and related two-dimensional plate models rely on the ability to approximate the deformation with a so-called Kirchhoff-Love displacement. This generally follows from an assumption that the in-plane components of the displacement are an order of magnitude smaller than the deflection, $u_{3}$. However, we find that even with $u_{1}$ and $u_{3}$ being of the same magnitude, the anisotropy allows us to approximate the displacement with a degenerate sort of Kirchhoff-Love displacement (see Eq. (21)), resulting in a robust bidimensional model.

In the anisotropic problem asymptotic expansions are not useful, since the resulting problem is over simplified. By isolating the terms in which different powers of $\epsilon$ appear, the expansions obscure the dependence of the deflection of the plate, $u_{3}$, upon $x_{1}$. So, rather than introduce asymptotic expansions, we construct functions that asymptotically approximate the solutions to the given problem, i.e., an approximate stress $\sigma^{0}$ and approximate displacement $\mathbf{u}^{0}$ that solve the three-dimensional problem up to an error of order $\epsilon$.

After the introduction of the approximate solutions, in Sec. 6 we reduce to two dimensions by considering the restriction of the approximating functions to the centerplane of the plate. The approximate displacement is shown to have degenerate Kirchhoff-Love form, and the approximate displacement $u_{1}^{0}$ is found to be independent of $x_{2}$. Finally, the approximating functions are constructed from their restrictions to the centerplane. The approximating nature of the construction is stated in a theorem at the end of the section; it is stated in terms of classical rather than weak solutions since the appropriate spaces for weak solutions are not clear. The boundary conditions for the deflection differ 
from the isotropic case (compare (4) with (7)) because of the degeneracy of the model, but these follow naturally from the three-dimensional conditions in this case. The construction of the degenerate boundary value problem described above is carried out in Sec. 7. The independence of $u_{1}^{0}$ from $x_{2}$ permits the construction of the equation for the Airy stress function that is uniformly elliptic under a mild restriction on the coefficients of the constitutive equations (see Eq. (15)).

The paper closes with some remarks on the model in Sec. 8. In particular, conditions that must be imposed in order to assure existence of solutions to the derived problem are presented. Further, difficulties in obtaining regularity results for boundary value problems of this type are mentioned, along with some modifications that may circumvent these obstacles.

4. Assumptions. We break our assumptions into three categories: the basic equilibrium assumptions of finite elasticity, the boundary conditions we impose on our deformation, and the constitutive assumptions that describe the "strong" anisotropy under consideration.

1. Equilibrium assumptions. We assume that the deformed state of the plate is in equilibrium with the forces described here. We assume the deformation does not alter the force densities given (dead loads). The only body force assumed to be acting on the plate is gravity (this could be generalized without difficulty). Its force density per unit volume will be denoted by $G^{\epsilon}:=\left(0,0,-g^{\epsilon}\right)$ defined on $\Omega^{\epsilon}$. We assume the boundary forces acting on the faces of the plate are normal to the reference plane: their density per unit area vector is $F^{\epsilon}:=\left(0,0, f^{\epsilon}\right)$ on $\Gamma_{ \pm}^{\epsilon}$. The boundary forces on $\Gamma_{0}^{\epsilon}$ are assumed to act only in the plane of the undeformed plate, and are uniformly distributed in height. Thus they can be given as a force density per unit length on $\gamma$ by $H^{\epsilon}:=\left(h_{1}^{\epsilon}, h_{2}^{\epsilon}, 0\right)$.

We use the standard equilibrium condition for nonlinear elasticity: The forces acting on the body are in equilibrium with the elastic forces described by the Piola-Kirchhoff stress tensor. The mathematical formulation consists of the following two conditions.

a) In the interior of $\Omega^{\epsilon}$ the elastic forces are given by the divergence of the PiolaKirchhoff stress tensor:

$$
G_{i}^{\epsilon}+\left(\sigma_{i j}+\sigma_{k j} u_{i, k}\right)_{, j}=0 \text { in } \Omega^{\epsilon} .
$$

b) On the boundary, the resultant force across the boundary is given by the dot product of the Piola-Kirchhoff stress tensor with the unit outer normal of the boundary. On $\Gamma_{ \pm}^{\epsilon}$ the equilibrium condition is:

$$
\left(\sigma_{i 3}+\sigma_{k 3} u_{i, k}\right) n_{3}=F_{i}^{\epsilon} \quad \text { on } \Gamma_{ \pm}^{\epsilon}
$$

On $\Gamma_{0}^{\epsilon}$ the condition can be written

$$
\frac{1}{2 \epsilon} \int_{-\epsilon}^{\epsilon}\left(\sigma_{i \beta}+\sigma_{j \beta} u_{i, j}\right) d x_{3} n_{\beta}=H_{i}^{\epsilon} \quad \text { on } \Gamma_{0}^{\epsilon} .
$$

2. Assumptions on the deformation/boundary conditions. The only assumptions we make on the nature of the deformation are the necessary boundary conditions on $\Gamma_{0}^{\epsilon}$. We 
use Ciarlet's conditions for a clamped plate:

$$
u_{\alpha} \text { independent of } x_{3} \text { for } \alpha=1,2 \text { and } u_{3}=0 \text { on } \Gamma_{0}^{\epsilon} .
$$

We assume the plate will not undergo any translation as a result of the forces imposed. Movement of the plate as a whole in the $x_{3}$ direction is prohibited by the boundary conditions above, but to avoid in-plane translation or rotation, we must impose constraints on the boundary forces:

$$
\int_{\gamma} h_{\alpha}^{\epsilon} d s=0 \quad \text { for } \alpha=1,2, \quad \text { and } \quad \int_{\gamma} h_{1}^{\epsilon} x_{2}-h_{2}^{\epsilon} x_{1} d s=0 .
$$

These conditions arise only in the introduction of the Airy stress function.

3. Constitutive assumptions. We use the usual Almansi strain tensor to describe the strain associated with the deformation $\mathbf{u}$ :

$$
\bar{\varepsilon}_{i j}(\mathbf{u}):=\frac{1}{2}\left(u_{i, j}+u_{j, i}+u_{k, i} u_{k, j}\right) .
$$

We assume a linear elastic constitutive law; however, we assume the material is "strongly" anisotropic, that is, the stiffness in one of the in-plane directions is "much less" than in the other principal directions. The simplest anisotropy (commonly called "transversely isotropic", see, e.g., [8]) that permits this phenomenon has the following generalized Hooke's laws:

$$
\begin{array}{ll}
\bar{\varepsilon}_{11}=\frac{1}{\epsilon \bar{E}}\left(\sigma_{11}-\nu \epsilon \sigma_{22}-\nu \epsilon \sigma_{33}\right), & \bar{\varepsilon}_{12}=\frac{1}{2 \bar{G}} \sigma_{12}, \\
\bar{\varepsilon}_{22}=\frac{1}{\bar{E}}\left(-\nu \sigma_{11}+\sigma_{22}-\bar{\nu} \sigma_{33}\right), & \bar{\varepsilon}_{13}=\frac{1}{2 \bar{G}} \sigma_{13}, \\
\bar{\varepsilon}_{33}=\frac{1}{\bar{E}}\left(-\nu \sigma_{11}-\bar{\nu} \sigma_{22}+\sigma_{33}\right), & \bar{\varepsilon}_{23}=\frac{1+\bar{\nu}}{\bar{E}} \sigma_{23} .
\end{array}
$$

The unbarred constants of the previous section are replaced by "constants" depending on $\epsilon: \bar{E}:=\left(1 / \epsilon^{3}\right) E$ is the Young's modulus in a plane of isotropy (parallel to the $\left(x_{2}, x_{3}\right)$ plane); $\epsilon \bar{E}$ is the Young's modulus in the $x_{1}$ direction; $\bar{G}:=\left(1 / \epsilon^{3}\right) G$ is the shear modulus across a plane of isotropy; and $\bar{\nu}$ and $\nu$ are the Poisson ratios, in and across a plane of isotropy, respectively. In the corrugated cardboard example suggested in the Introduction, this loss of stiffness in the $x_{1}$ direction corresponds to the corrugations running parallel to the $x_{2}$-axis. The introduction of the factor $\epsilon^{-3}$ formalizes a physical notion arrived at by comparing plates of various thicknesses under identical loads. A thinner plate must be stiffer than a thicker one to undergo a deformation of the same magnitude. So, we introduce the heuristically chosen weight here, as we do not wish the solution to our problem to change magnitude as $\epsilon$ grows small.

The laws given assume a hyperelastic material; the coefficients of the equations are symmetric. Notice that the contributions of $\sigma_{22}$ and $\sigma_{33}$ to the strain $\bar{\varepsilon}_{11}$ are of the order $O\left(\epsilon^{-1}\right)$, while the contribution of $\sigma_{11}$ to the strains $\bar{\varepsilon}_{22}$ and $\bar{\varepsilon}_{33}$ is of the order $O(1)$. It is possible for hyperelastic constitutive relations to have the strong anisotropy stipulated here, but with Poisson ratios of different magnitudes. The relations above can be modified by replacing $\nu$ with $\nu \epsilon^{-r}$. For $0 \leq r \leq 1$, the treatment below obtains, with modifications. A similar statement can be made about the magnitude of the shear modulus $G$. 
For technical reasons we require that

$$
\nu<4 G^{2}+E .
$$

This condition assures the ellipticity of the equation governing the Airy stress function. We will abbreviate Eq. (14) by $\bar{\varepsilon}_{i j}=(A \sigma)_{i j}$, where $A$ can be thought of as the fourthorder tensor of coefficients $\left(\bar{\varepsilon}_{i j}=A^{i j k l} \sigma_{k l}\right)$. We also assume that all data is square integrable on its domain of definition, that is,

$$
g^{\epsilon} \in L^{2}\left(\Omega^{\epsilon}\right), \quad h_{\alpha}^{\epsilon} \in L^{2}(\gamma), \quad F_{i}^{\epsilon} \in L^{2}\left(\Gamma_{ \pm}^{\epsilon}\right) .
$$

5. Formulation of the three-dimensional problem. We translate the problem Eqs. (8), (9), (10), (11), and (14) into a weak problem on a domain independent of epsilon. We begin the weak formulation by introducing the following spaces of test functions. The test space for tensors is

$$
\Sigma^{\epsilon}:=\left\{L^{2}\left(\Omega^{\epsilon}\right)\right\}_{\mathrm{symm}}^{3 \times 3},
$$

that is, the space of all symmetric three by three matrices with square integrable entries. For deformations we use the space

$$
V^{\epsilon}:=\left\{\mathbf{v} \in\left\{W^{(1,4)}\left(\Omega^{\epsilon}\right)\right\}^{3}: \mathbf{v} \text { satisfies }(11)\right\}
$$

in other words, the space of $W^{(1,4)}$ vector fields on $\Omega^{\epsilon}$ satisfying the boundary conditions (11), in the sense of trace.

If $\mathbf{u}$ and the stress $\sigma$ solve Eq. (14), then

$$
\int_{\Omega^{\epsilon}}\left(u_{i, j}+\frac{1}{2} u_{k, i} u_{k, j}\right) \tau_{i j}-(A \sigma)_{i j} \tau_{i j} d V=0 \quad \text { for all } \tau \in \Sigma^{\epsilon},
$$

and solutions $\mathbf{u}$ and $\sigma$ of this equation need only belong to $V^{\epsilon}$ and $\Sigma^{\epsilon}$, respectively. By integrating Eq. (8) against $\mathbf{v} \in V^{\epsilon}$, applying the divergence theorem, and using Eqs. (9) and (10) to substitute for boundary values, we arrive at

$$
\begin{aligned}
\int_{\Omega^{\epsilon}}( & \left.\sigma_{i j}+\sigma_{k j} u_{i, k}\right) v_{i, j} d V \\
& =-\int_{\Omega^{\epsilon}} g^{\epsilon} v_{3} d V+\int_{\Gamma_{ \pm}^{\epsilon}} f^{\epsilon} v_{3} d A+\int_{\gamma} h_{\alpha}^{\epsilon}\left(\int_{-\epsilon}^{\epsilon} v_{\alpha} d x_{3}\right) d x \quad \text { for all } \mathbf{v} \in V^{\epsilon},
\end{aligned}
$$

and this formulation also makes sense for $\mathbf{u} \in V^{\epsilon}, \sigma \in \Sigma^{\epsilon}$.

We convert the weak formulation of the problem (16), (17), with boundary conditions (11), into a problem on $\Omega:=\Omega^{1}=\omega \times(-1,1)$, with boundary components $\Gamma_{0}:=\Gamma_{0}^{1}$, $\Gamma_{+}:=\Gamma_{+}^{1}, \Gamma_{-}:=\Gamma_{-}^{1}$, and $\Gamma_{ \pm}:=\Gamma_{ \pm}^{1}$. We introduce the spaces $V:=V^{1}, \Sigma:=\Sigma^{1}$, and the linear bijection between $\bar{\Omega}$ and $\bar{\Omega}^{\epsilon}$ given by associating any $x:=\left(x_{1}, x_{2}, \chi\right) \in \Omega$ to $x^{\epsilon}=\left(x_{1}, x_{2}, \epsilon \chi\right) \in \Omega^{\epsilon}$. This correspondence induces the following transformations of vector fields and symmetric tensors from the domain $\Omega^{\epsilon}$ to $\Omega$. For any vector field $\mathbf{v}$ defined on $\Omega^{\epsilon}$ we can construct a $\mathbf{v}^{\epsilon}$ defined on $\Omega$ so that

$$
v_{2}\left(x^{\epsilon}\right)=\epsilon^{2} v_{2}^{\epsilon}(x): \quad v_{i}\left(x^{\epsilon}\right)=\epsilon v_{i}^{\epsilon}(x) \quad \text { for } i=1,3 .
$$


Similarly, any symmetric tensor $\tau$ with domain $\Omega^{\epsilon}$ can be expressed in terms of a corresponding tensor $\tau^{\epsilon}$ on $\Omega$ using the weights

$$
\begin{gathered}
\tau_{\alpha \beta}\left(x^{\epsilon}\right)=\frac{1}{\epsilon} \tau_{\alpha \beta}^{\epsilon}(x) \quad \text { for } \alpha, \beta=1,2 ; \quad \tau_{\alpha 3}\left(x^{\epsilon}\right)=\tau_{\alpha 3}^{\epsilon}(x) \quad \text { for } \alpha=1,2 \\
\tau_{33}\left(x^{\epsilon}\right)=\epsilon \tau_{33}^{\epsilon}(x) .
\end{gathered}
$$

These weights are based on those used in [2], as mentioned in Sec. 3. We assume that the various components of the solutions of the transformed problem, $\mathbf{u}^{\epsilon}$ and $\sigma^{\epsilon}$ are of comparable magnitudes. Notice, this is an implicit assumption about the relative magnitudes of the original displacement's and stress's components. For instance, assuming that $u_{2}^{\epsilon}=\frac{1}{\epsilon^{2}} u_{2}$ is comparable in size to $u_{3}^{\epsilon}=\frac{1}{\epsilon} u_{3}$ is an assertion that $u_{2}$ is smaller than $u_{3}$ by a factor of $\epsilon$. Under the change in variables, integrals in $\Omega^{\epsilon}$ are equal to the integrals of the corresponding functions in $\Omega$ provided we use the appropriate differentials.

We now apply this change of coordinates to Eq. (16). Recall $\bar{E}=E / \epsilon^{3}$ and $\bar{G}=G / \epsilon^{3}$. Dividing through by $\epsilon^{2}$, we arrive at

$$
\begin{aligned}
\int_{\Omega} \frac{1}{E}[ & \left.\tau_{11}^{\epsilon}\left(\frac{1}{\epsilon} \sigma_{11}^{\epsilon}-\nu \sigma_{22}^{\epsilon}\right)+\tau_{22}^{\epsilon}\left(\sigma_{22}^{\epsilon}-\nu \sigma_{11}^{\epsilon}\right)\right] \\
& +\frac{1}{2 G}\left(\tau_{12}^{\epsilon} \sigma_{12}^{\epsilon}+\tau_{21}^{\epsilon} \sigma_{21}^{\epsilon}\right)-\epsilon^{2} \mathcal{B}_{2}\left(\tau^{\epsilon}, \sigma^{\epsilon}\right)-\epsilon^{4} \tau_{33}^{\epsilon} \sigma_{33}^{\epsilon} d V \\
= & \int_{\Omega} \tau_{i j}^{\epsilon}\left[\frac{1}{\epsilon_{i}^{1}} u_{i, j}^{\epsilon}+\frac{1}{2}\left(u_{1, i}^{\epsilon} u_{1, j}^{\epsilon}+u_{3, i}^{\epsilon} u_{3, j}^{\epsilon}\right)+\frac{\epsilon^{2}}{2} u_{2, i}^{\epsilon} u_{2, j}^{\epsilon}\right] d V
\end{aligned}
$$

for all $\tau \in \Sigma$, where the bilinear form is

$$
\begin{aligned}
\mathcal{B}_{2}(\tau, \sigma)= & \frac{\nu}{E}\left(\tau_{11} \sigma_{33}+\sigma_{11} \tau_{33}\right)+\frac{\bar{\nu}}{E}\left(\tau_{22} \sigma_{33}+\sigma_{22} \tau_{33}\right) \\
& -\frac{1}{2 G}\left(\tau_{13} \sigma_{13}+\tau_{31} \sigma_{31}\right)-\frac{1+\bar{\nu}}{E}\left(\tau_{23} \sigma_{23}+\tau_{32} \sigma_{32}\right),
\end{aligned}
$$

and $\epsilon_{i}^{1}$ is defined by

$$
\epsilon_{i}^{1}= \begin{cases}\epsilon & \text { if } i=1, \\ 1 & \text { otherwise }\end{cases}
$$

We now treat Eq. (17) in the same way; first defining analogues of $g^{\epsilon}, f^{\epsilon}$, and $h_{\alpha}^{\epsilon}$ on $\Omega$ by

$$
g^{\epsilon}\left(x^{\epsilon}\right)=g(x), \quad f^{\epsilon}\left(x^{\epsilon}\right)=\epsilon f(x), \quad h_{\alpha}^{\epsilon}\left(x^{\epsilon}\right)=\frac{1}{\epsilon} h_{\alpha}(x) .
$$

The new functions $h_{\alpha}$ will satisfy the conditions (12), since $h_{\alpha}^{\epsilon}$ did. Converting the equilibrium equation (17) into its corresponding equation on $\Omega$, we arrive at

$$
\begin{aligned}
\int_{\Omega} \sigma_{i j}^{\epsilon} & \left(\frac{1}{\epsilon_{i}^{1}} v_{i, j}^{\epsilon}+u_{1, i}^{\epsilon} v_{1, j}^{\epsilon}+u_{3, i}^{\epsilon} v_{3, j}^{\epsilon}+\epsilon^{2} u_{2, i}^{\epsilon} v_{2, j}^{\epsilon}\right) d V \\
= & -\int_{\Omega} g v_{3}^{\epsilon} d V+\int_{\Gamma_{ \pm}} f v_{3}^{\epsilon} d A \\
& +\int_{\gamma} \frac{1}{\epsilon} h_{1} \int_{-1}^{1} v_{1}^{\epsilon} d \chi+h_{2} \int_{-1}^{1} v_{2}^{\epsilon} d \chi d s \quad \text { for all } \mathbf{v} \in V
\end{aligned}
$$


The weak formulation and change of variables just completed places all dependence of the problem upon $\epsilon$ in the solutions, $\sigma^{\epsilon} \in \Sigma$ and $\mathbf{u}^{\epsilon} \in V$. We now introduce a twodimensional problem from whose solutions we can reconstruct approximations to the solutions of this three-dimensional problem. The asymptotic nature of this approximate solution to the given problem is: the approximating functions solve the three-dimensional problem up to an error $O(\epsilon)$. The approximations will be denoted $\mathbf{u}^{0}$ and $\sigma^{0}$.

6. Derivation of the two-dimensional problem. From the three-dimensional problem Eqs. (18), (19), with boundary conditions (11) imposed on $\mathbf{u}^{\epsilon}$, we wish to construct the two-dimensional problem, which we can then convert to the system of equations presented in Sec. 3. We begin by introducing the restrictions of the three-dimensional approximations to the center-plane of the plate:

$$
\bar{u}_{i}\left(x_{1}, x_{2}\right):=u_{i}^{0}\left(x_{1}, x_{2}, 0\right) \quad \text { and } \quad \bar{\sigma}_{i j}\left(x_{1}, x_{2}\right):=\sigma_{i j}^{0}\left(x_{1}, x_{2}, 0\right) .
$$

Now we express the three-dimensional approximations in terms of the two-dimensional functions, and find an appropriate bidimensional problem that these new functions solve. Notice that Eq. (18), by considering test functions for which $\tau_{13}=\tau_{31}$ are the only nonzero entries, implies

$$
u_{1,3}^{\epsilon}+\epsilon\left(u_{3,1}^{\epsilon}+u_{1,3}^{\epsilon} u_{1,1}^{\epsilon}+u_{3,1}^{\epsilon} u_{3,3}^{\epsilon}\right)=O\left(\epsilon^{3}\right) \text { a.e. in } \Omega
$$

after multiplying through by $\epsilon$. Solving for $u_{1,3}^{\epsilon}$, and applying the expansion

$$
\frac{1}{1+\epsilon u_{1,1}^{\epsilon}}=\sum_{k=0}^{\infty}\left(-\epsilon u_{1,1}^{\epsilon}\right)^{k}
$$

(which holds formally for $\epsilon$ sufficiently small) we find

$$
u_{1,3}^{\epsilon}=-\frac{\epsilon u_{3,1}^{\epsilon}\left(1+u_{3,3}^{\epsilon}\right)}{1+\epsilon u_{1,1}^{\epsilon}}+O\left(\epsilon^{3}\right)=-\epsilon u_{3,1}^{\epsilon}\left(1+u_{3,3}^{\epsilon}\right)+O\left(\epsilon^{2}\right) .
$$

In short, it must be that $u_{1,3}^{\epsilon}=O(\epsilon)$. If we return to Eq. (18), inserting test functions for which only $\tau_{33}$ is nonzero gives

$$
u_{3,3}^{\epsilon}\left(2+u_{3,3}^{\epsilon}\right)=-\left(u_{1,3}^{\epsilon}\right)^{2}+O\left(\epsilon^{2}\right)=O\left(\epsilon^{2}\right)
$$

by (20). Thus we require of our approximating function, $u_{33}^{0}=0$ or $u_{3,3}^{0}=-2$ a.e. in $\Omega$. Assuming $u_{3,3}^{0}$ is continuous (and not merely in $L^{4}(\Omega)$ ), the boundary conditions (11) imply that it must vanish in $\bar{\Omega}$ (since it is zero on $\Gamma_{0}$ ). So, $u_{3}^{0}$ is constant in the $x_{3}$ direction, or $u_{3}^{0}\left(x_{1}, x_{2}, x_{3}\right)=\bar{u}_{3}\left(x_{1}, x_{2}\right)$. Using the fact that $u_{3,3}^{\epsilon}=O\left(\epsilon^{2}\right)$ in Eq. (20) we have $u_{1,3}^{\epsilon}=-\epsilon u_{3,1}^{\epsilon}+O\left(\epsilon^{2}\right)$, which permits us to assume that $u_{1,3}^{0}=-\epsilon \bar{u}_{3,1}$. By inserting test functions with only $\tau_{23}=\tau_{32}$ nonzero in (18), we find that $u_{2,3}^{0}=-\bar{u}_{3,2}$ by analogous reasoning. So, the three-dimensional displacement $\mathbf{u}^{\epsilon}$ can be asymptotically approximated with error $O\left(\epsilon^{2}\right)$ by a "degenerate" Kirchhoff-Love displacement satisfying

$$
\begin{aligned}
& u_{1}^{0}\left(x_{1}, x_{2}, x_{3}\right)=\bar{u}_{1}\left(x_{1}, x_{2}\right)-\epsilon x_{3} \bar{u}_{3,1}\left(x_{1}, x_{2}\right), \\
& u_{2}^{0}\left(x_{1}, x_{2}, x_{3}\right)=\bar{u}_{2}\left(x_{1}, x_{2}\right)-x_{3} \bar{u}_{3,2}\left(x_{1}, x_{2}\right), \\
& u_{3}^{0}\left(x_{1}, x_{2}, x_{3}\right)=\bar{u}_{3}\left(x_{1}, x_{2}\right) .
\end{aligned}
$$


We will eventually take $u_{1}^{0}\left(x_{1}, x_{2}, x_{3}\right)=\bar{u}_{1}\left(x_{1}, x_{2}\right)$ as our approximating function, since we regard $\epsilon$ as asymptotically small. We use the form in Eq. (21) through the derivation of the in-plane stress components (where an $\epsilon^{-1} u_{1,2}^{\epsilon}$ term appears). Thus, we have in the end approximated $u_{1}^{\epsilon}$ only up to order $\epsilon$.

Further, applying the boundary conditions (11) to the approximate displacement, we have $u_{\alpha, 3}^{0}=0$ on $\Gamma_{0}$ (in the sense of trace); so $u_{3,2}^{0}=\bar{u}_{3,2}=0$, as well as $u_{3}^{0}=\bar{u}_{3}=0$ on $\Gamma_{0}$ or $\gamma$ as appropriate. Since $\epsilon$ is considered asymptotically small, the boundary conditions give us no control over $\bar{u}_{3,1}$ on $\gamma$. Instead, knowing that $\bar{u}_{3} \equiv 0$ on $\gamma$ we use that its tangential derivative, $d \bar{u}_{3} / d s$, vanishes there also. Thus we have

$$
0=\frac{d \bar{u}_{3}}{d s}=-n_{2} \bar{u}_{3,1}+n_{1} \bar{u}_{3,2}=-n_{2} \bar{u}_{3,1}
$$

since $\bar{u}_{3,2}=0$ on $\gamma$. So, $\bar{u}_{3,1}=0$ on portions of the boundary where $n_{2} \neq 0$. Our boundary conditions on $\bar{u}_{3}$ can be written:

$$
\begin{gathered}
\bar{u}_{3}=0 \quad \text { on all of } \gamma, \\
\frac{\partial \bar{u}_{3}}{\partial \mathbf{n}}=0 \quad \text { on } \gamma \text { where } n_{2} \neq 0 .
\end{gathered}
$$

These are precisely the boundary conditions necessary for a well-posed problem in the linear case for an equation of the form (5).

Now, we use the cases where all entries but a fixed $\tau_{\alpha \beta}$ in (18) vanish to express the approximate in-plane stress, $\sigma_{\alpha \beta}^{0}$, in terms of $\overline{\mathbf{u}}$. Direct substitution yields weak forms of

$$
\begin{aligned}
\frac{1}{\epsilon} \sigma_{11}^{\epsilon}-\nu \sigma_{22}^{\epsilon} & \left.=E\left[\frac{1}{\epsilon} u_{1,1}^{\epsilon}+\frac{1}{2}\left(u_{1,1}^{\epsilon}\right)^{2}+\left(u_{3,1}^{\epsilon}\right)^{2}\right)\right]+O\left(\epsilon^{2}\right), \\
-\nu \sigma_{11}^{\epsilon}+\sigma_{22}^{\epsilon} & \left.=E\left[u_{2,2}^{\epsilon}+\frac{1}{2}\left(u_{1,2}^{\epsilon}\right)^{2}+\left(u_{3,2}^{\epsilon}\right)^{2}\right)\right]+O\left(\epsilon^{2}\right), \\
\sigma_{12}^{\epsilon} & =G\left[\frac{1}{\epsilon} u_{1,2}^{\epsilon}+u_{2,1}^{\epsilon}+u_{1,1}^{\epsilon} u_{1,2}^{\epsilon}+u_{3,1}^{\epsilon} u_{3,2}^{\epsilon}\right]+O\left(\epsilon^{2}\right) .
\end{aligned}
$$

So that we may substitute $\mathbf{u}^{0}$ for $\mathbf{u}^{\epsilon}$ as defined in (21) into these equations, we must assume more regularity on $\bar{u}_{3}$, namely $\bar{u}_{3} \in H^{2}(\omega)$. Making this assumption and substitution will lower the order of the error in the first and third of these equations to $O(\epsilon)$. Solving the third equation above (after substitution) for $u_{1,2}^{0}$, we find that it is $O(\epsilon)$. Since this is the order of $u_{1}^{0}$ 's approximation to $u_{1}^{\epsilon}$, we assume that $0=u_{1,2}^{0}\left(x_{1}, x_{2}, x_{3}\right)=$ $\bar{u}_{1,2}\left(x_{1}, x_{2}\right)$, i.e., $\bar{u}_{1}$ depends only on $x_{1}$. Using this fact, and Eq. (21), we find that the stresses $\sigma_{\alpha \beta}^{\epsilon}$ are approximated up to order $\epsilon$ by linear functions of $x_{3}$. We are led to define $\sigma_{\alpha \beta}^{0}=\bar{\sigma}_{\alpha \beta}+x_{3} 3 m_{\alpha \beta}$ with the coefficients

$$
\begin{array}{lll}
\bar{\sigma}_{11}:=E \bar{u}_{1,1}, & m_{11}:=0, \\
\bar{\sigma}_{22}:=E\left[\bar{u}_{2,2}+\nu \bar{u}_{1,1}+\frac{1}{2}\left(\bar{u}_{3,2}\right)^{2}\right], & m_{22}:=-\frac{E}{3} \bar{u}_{3,22}, \\
\bar{\sigma}_{12}:=G\left(\bar{u}_{2,1}+\bar{u}_{3,1} \bar{u}_{3,2}\right), & m_{12}:=-\frac{2 G}{3} \bar{u}_{3,12} .
\end{array}
$$

Notice that these equations make sense for $\overline{\mathbf{u}}$ satisfying $\bar{u}_{\alpha} \in H^{1}(\omega), \bar{u}_{3} \in W^{(1,4)}(\omega)$, and $\bar{u}_{3,2} \in H^{1}(\omega)$. Further, the boundary conditions (22) hold in the sense of trace for all $\bar{u}_{3}$ 
in the closure of $C_{0}^{\infty}(\omega)$ under the norm $\|v\|_{W^{(1,4)}(\omega)}+\left\|v_{, 2}\right\|_{H^{1}(\omega)}$; call this Banach space $U$, and define

$$
\mathcal{U}=\left\{\mathbf{u}: u_{\alpha} \in H^{1}(\omega) \text { for } \alpha=1,2, \text { and } u_{3} \in U\right\}
$$

We will see that this is the appropriate space for solutions of the two-dimensional problem. Now consider $\tau \in \Sigma$, a test function; decompose it into its even and odd parts

$$
\begin{aligned}
& \tau_{\alpha \beta}^{e}\left(x_{1}, x_{2}, x_{3}\right):=\frac{1}{2}\left(\tau_{\alpha \beta}\left(x_{1}, x_{2}, x_{3}\right)+\tau_{\alpha \beta}\left(x_{1}, x_{2},-x_{3}\right)\right), \\
& \tau_{\alpha \beta}^{o}\left(x_{1}, x_{2}, x_{3}\right):=\frac{1}{2}\left(\tau_{\alpha \beta}\left(x_{1}, x_{2}, x_{3}\right)-\tau_{\alpha \beta}\left(x_{1}, x_{2},-x_{3}\right)\right),
\end{aligned}
$$

with average values defined by

$$
\begin{aligned}
& \bar{\tau}_{\alpha \beta}^{e}\left(x_{1}, x_{2}\right):=\frac{1}{2} \int_{-1}^{1} \tau_{\alpha \beta}^{e}\left(x_{1}, x_{2}, x_{3}\right) d x_{3}, \\
& \bar{\tau}_{\alpha \beta}^{o}\left(x_{1}, x_{2}\right):=\frac{3}{2} \int_{-1}^{1} x_{3} \tau_{\alpha \beta}^{o}\left(x_{1}, x_{2}, x_{3}\right) d x_{3} .
\end{aligned}
$$

Using this decomposition, and the linearity of the in-plane stress tensor in $x_{3}$, yields

$$
\begin{aligned}
& \int_{\Omega} \tau_{\alpha \beta}\left(x_{1}, x_{2}, x_{3}\right) \sigma_{\alpha \beta}^{0}\left(x_{1}, x_{2}, x_{3}\right) d V \\
& \quad=2 \int_{\omega} \bar{\tau}_{\alpha \beta}^{e}\left(x_{1}, x_{2}\right) \bar{\sigma}_{\alpha \beta}\left(x_{1}, x_{2}\right) d A+2 \int_{\omega} \bar{\tau}_{\alpha \beta}^{o}\left(x_{1}, x_{2}\right) m_{\alpha \beta}\left(x_{1}, x_{2}\right) d A .
\end{aligned}
$$

Thus, Eq. (18), with the approximate stress and displacement substituted, has an equivalent statement in two dimensions, and the given approximations satisfy it up to $O(\epsilon)$.

To determine equilibrium conditions imposed on the bidimensional functions, $\overline{\mathbf{u}}, \bar{\sigma}_{\alpha \beta}$, and $m_{\alpha \beta}$, insert a test function whose only nonzero entry is $v_{1}\left(x_{1}, x_{2}, x_{3}\right)=\bar{v}\left(x_{1}, x_{2}\right)$ into (19), to find

$$
\int_{\Omega} \sigma_{11}^{\epsilon}\left(\frac{1}{\epsilon} \bar{v}_{, 1}+u_{1,1}^{\epsilon} \bar{v}_{, 1}\right)+\sigma_{12}^{\epsilon}\left(\frac{1}{\epsilon} \bar{v}_{, 2}+u_{1,1}^{\epsilon} \bar{v}_{, 2}\right) d V=2 \int_{\gamma} \frac{h_{1}}{\epsilon} \bar{v} d s .
$$

Here $\bar{v}$ can be any $H^{1}(\omega)$ function. Multiplying through by $\epsilon$, and substituting the approximating functions for $\mathbf{u}^{\epsilon}$ and $\sigma^{\epsilon}$, we arrive at

$$
\int_{\Omega} \sigma_{1 \alpha}^{0} \bar{v}_{, \alpha} d V=2 \int_{\gamma} h_{1} \bar{v} d s+O(\epsilon) .
$$

We require this equality to hold exactly, that is, without the order $\epsilon$ error. Using the form for $\sigma^{0}$ given by Eq. (23), integrate out the $x_{3}$ variable to arrive at

$$
\int_{\omega} \bar{\sigma}_{\beta \alpha} \bar{v}_{, \alpha} d A=\int_{\gamma} h_{\beta} \bar{v} d s
$$

for $\beta=1$. The proof for $\beta=2$ is analogous. Notice that this equation makes sense not only for $\bar{v} \in W^{(1,4)}(\omega)$, as $v \in V$ would imply, but for $\bar{v}$ in the larger space $\bar{v} \in H^{1}(\omega)$. This is the space of test functions that we will use in formulating our two-dimensional problem. Notice that it follows from the definitions (23) that this equation makes sense even if $u_{\alpha} \in H^{1}(\omega)$ so long as $u_{3} \in W^{(1,4)}(\omega)$.

Define a space of test functions on $\omega$ by

$$
\bar{V}=\left\{\bar{v} \in W^{(2,4)}(\omega):(22) \text { holds for } \bar{u}_{3}=\bar{v}\right\}
$$


Inserting into (19) those test functions with the only nonzero entry $v_{1}\left(x_{1}, x_{2}, x_{3}\right)=$ $-x_{3} \bar{v}_{, 1}\left(x_{1}, x_{2}\right)$ for some $\bar{v} \in \bar{V}$, we have

$$
\int_{\Omega}-x_{3}\left(\sigma_{11}^{\epsilon} \bar{v}_{, 11}+\sigma_{12}^{\epsilon} \bar{v}_{, 12}\right)\left(\frac{1}{\epsilon}+u_{1,1}^{\epsilon}\right)-\sigma_{13}^{\epsilon} \bar{v}_{, 1}\left(\frac{1}{\epsilon}+u_{1,1}^{\epsilon}\right) d V=0 .
$$

Multiplying through by $\epsilon$, and replacing the $\sigma_{\alpha \beta}^{\epsilon}$ with their approximations we find that

$$
\int_{\Omega} \sigma_{13}^{\epsilon} \bar{v}_{, 1} d V=\int_{\Omega}-x_{3} \sigma_{1 \alpha}^{0} \bar{v}_{, 1 \alpha} d V+O(\epsilon)
$$

We require this equation to hold exactly for the approximation $\sigma_{13}^{0}$, and by integrating out the $x_{3}$ on the right-hand side find

$$
\int_{\Omega} \sigma_{\beta 3}^{0} \bar{v}_{, \beta} d V=-2 \int_{\omega} m_{\beta \alpha} \bar{v}_{, \beta \alpha} d A \quad \text { for all } \bar{v} \in \bar{V}
$$

for $\beta=1$, but again the equation follows in a similar fashion for $\beta=2$.

We now substitute $\mathbf{v}^{\epsilon}=\left(0,0, \bar{v}\left(x_{1}, x_{2}\right)\right)$, and $\mathbf{u}^{0}$ and $\sigma^{0}$ for $\mathbf{u}^{\epsilon}$ and $\sigma^{\epsilon}$ in Eq. (19), with $\bar{v}$ from $\bar{V}$. Defining

$$
\bar{f}\left(x_{1}, x_{2}\right):=\frac{1}{2}\left(-\int_{-1}^{1} g\left(x_{1}, x_{2}, x_{3}\right) d x_{3}+f\left(x_{1}, x_{2}, 1\right)+f\left(x_{1}, x_{2},-1\right)\right),
$$

and using (25) to eliminate the $\sigma_{3 \alpha}^{0}$ terms, we have that, up to an error of order $\epsilon$,

$$
\int_{\omega}-m_{\alpha \beta} \bar{v}_{, \alpha \beta}+\bar{\sigma}_{\alpha \beta} \bar{u}_{3, \alpha} \bar{v}_{, \beta} d A=\int_{\omega} \bar{f} \bar{v} d A
$$

for all $\bar{v} \in \bar{V}$; but we require this equation to hold exactly for our two-dimensional approximating functions.

We require: Eq. (23) to define $\bar{\sigma}$ and $m$; Eq. (24) to hold for any $\bar{v} \in H^{1}(\omega)$; and Eq. (26) to hold for any $\bar{v} \in U$ for some $\mathbf{u} \in \mathcal{U}$. This is the two-dimensional problem approximating the original three-dimensional problem given by Eqs. (18), (19), with boundary conditions (11). It is clear that we can construct the approximating functions $\mathbf{u}^{0}$ and $\sigma_{\alpha \beta}^{0}$ from the solutions $\overline{\mathbf{u}}, \bar{\sigma}$, and $m$, using Eq. (21) and the definitions of $\bar{\sigma}$ and $m$ (just before Eq. (23)). To complete the approximation, it remains to construct $\sigma_{i 3}^{0}$ (for $i=1,2,3$ ) in terms of $\overline{\mathbf{u}}$. To accomplish this, we must assume more regularity on $\bar{u}_{3}$. We specify the further assumptions in the statement of the theorem following the constructions.

To construct the $\sigma_{\alpha 3}^{0}$, we substitute into Eq. (19) $\mathbf{v}$ from $V$ with only $v_{1}=v$ nonzero. Decomposing $v$ into $v^{e}$ and $v^{o}$ as we did with $\tau$ above, we note that (11) implies

$$
\left.\begin{array}{l}
\bar{v}^{e}\left(x_{1}, x_{2}\right)=v^{e}\left(x_{1}, x_{2}, x_{3}\right)=v \quad \text { and } \\
\bar{v}^{o}\left(x_{1}, x_{2}\right)=v^{o}\left(x_{1}, x_{2}, x_{3}\right) \equiv 0
\end{array}\right\} \quad \text { on } \gamma \text { or } \Gamma_{0} \text { as appropriate. }
$$

Thus (19) yields

$$
\int_{\Omega} \sigma_{13}^{\epsilon} v_{, 3} d V=-\int_{\Omega} \sigma_{1 \alpha}^{\epsilon} v_{, \alpha} d V+\int_{\gamma} h_{1} \int_{-1}^{1} v d x_{3} d s+O(\epsilon) .
$$

If we require this equation to hold without the order $\epsilon$ error for the approximating functions $\sigma_{1 j}^{0}$, then we can integrate out the $x_{3}$ variable on the right-hand side to arrive 
at:

$$
\int_{\Omega} \sigma_{13}^{0} v_{, 3} d V=-2 \int_{\omega} \bar{\sigma}_{1 \alpha} \bar{v}_{, \alpha}^{e}+m_{1 \alpha} \bar{v}_{, \alpha}^{o} d A+2 \int_{\gamma} h_{1} \bar{v}^{e} d s .
$$

By Eq. (24), the area integral against $\bar{v}^{e}$ and the boundary integral cancel, and assuming $m$ is sufficiently regular we can apply the divergence theorem to show

$$
\begin{aligned}
\int_{\Omega} \sigma_{13}^{0} v_{, 3} d V & =-3 \int_{\Omega} x_{3} m_{1 \alpha}\left(x_{1}, x_{2}\right) \frac{1}{2}\left(v_{, \alpha}\left(x_{1}, x_{2}, x_{3}\right)-v_{, \alpha}\left(x_{1}, x_{2},-x_{3}\right)\right) d V \\
& =3 \int_{\Omega} x_{3} m_{1 \alpha, \alpha}\left(x_{1}, x_{2}\right) v\left(x_{1}, x_{2}, x_{3}\right) d V
\end{aligned}
$$

A similar result can be derived for $\sigma_{23}^{\epsilon}$. Since this is true for all $v \in C_{0}^{\infty}(\Omega)$ (this is a subset of $V$ ), we have that $\sigma_{\beta 3,3}^{0}\left(x_{1}, x_{2}, x_{3}\right)=-3\left[x_{3} m_{\beta \alpha, \alpha}\left(x_{1}, x_{2}\right)\right]$ on $\Omega$. Applying the divergence theorem to the left side of $(28)$ for test functions that include $\Gamma_{ \pm}$in their support, we see that $\sigma_{\beta 3}^{0} \equiv 0$ on $\Gamma_{ \pm}$. Thus, we have

$$
\sigma_{\beta 3}^{0}\left(x_{1}, x_{2}, x_{3}\right)=-3 \int_{-1}^{x_{3}} \chi m_{\beta \alpha, \alpha}\left(x_{1}, x_{2}\right) d \chi=\frac{3}{2}\left(1-x_{3}^{2}\right) m_{\beta \alpha, \alpha}\left(x_{1}, x_{2}\right) .
$$

This leaves only $\sigma_{33}^{0}$ to be computed. We begin by inserting a test function $\mathbf{v} \in V$ into Eq. (19) whose only nonzero entry is $v_{3}=v$ satisfying $\left.v\right|_{\Gamma_{0}} \equiv 0$. Isolating the $\sigma_{33}^{\epsilon}$ term, we have

$$
\int_{\Omega} \sigma_{33}^{\epsilon} v_{, 3} d V=\int_{\Omega} \sigma_{3 \alpha}^{\epsilon} v_{, \alpha}+\sigma_{\alpha j}^{\epsilon} u_{3, \alpha}^{\epsilon} v_{, j}-g v d V+\int_{\Gamma_{ \pm}} f v d A+O(\epsilon) .
$$

Using $\sigma^{0}$ and $\mathbf{u}^{0}$ in place of the solutions on the right-hand side does not increase the order of the error; so we require that the approximating function $\sigma_{33}^{0}$ satisfies

$$
\int_{\Omega} \sigma_{33}^{0} v_{, 3} d V=\int_{\Omega}\left[\sigma_{3 \alpha, \alpha}^{0}+\left(\sigma_{\alpha j}^{0} u_{3, \alpha}^{0}\right)_{, j}-g\right] v d V+\int_{\Gamma_{ \pm}} f v d A
$$

which follows from the substitution via the divergence theorem, using that $\sigma_{\alpha 3}^{0} \equiv 0$ on $\Gamma_{ \pm}$. So,

$$
\begin{aligned}
\sigma_{33,3}^{0} & =-\left[\sigma_{3 \alpha, \alpha}^{0}+\sigma_{\alpha 3,3}^{0} u_{3, \alpha}^{0}+\left(\sigma_{\alpha \beta}^{0} u_{3, \alpha}^{0}\right)_{, \beta}-g\right] \text { in } \Omega, \\
\sigma_{33}^{0} & = \pm f \quad \text { on } \Gamma_{+} \text {and } \Gamma_{-}, \text {respectively. }
\end{aligned}
$$

Substitute into the first equation above Eq. (29) and the definition of $\bar{\sigma}$ and $m$ to find

$$
\sigma_{33,3}^{0}=-\left[\frac{3}{2} m_{\alpha \beta, \alpha \beta}\left(1-x_{3}^{2}\right)+3 x_{3} m_{\alpha \beta} \bar{u}_{3, \alpha \beta}-\left(\bar{\sigma}_{\alpha \beta} \bar{u}_{3, \alpha}\right)_{, \beta}\right] .
$$

By substituting this into the trivial identity

$$
\begin{array}{r}
\sigma_{33}^{0}\left(x_{1}, x_{2}, x_{3}\right)=\frac{1}{2}\left(\sigma_{33}^{0}\left(x_{1}, x_{2},-1\right)+\sigma_{33}^{0}\left(x_{1}, x_{2}, 1\right)+\int_{-1}^{x_{3}} \sigma_{33,3}^{0}\left(x_{1}, x_{2}, \chi\right) d \chi\right. \\
\left.-\int_{x_{3}}^{1} \sigma_{33,3}^{0}\left(x_{1}, x_{2}, \chi\right) d \chi\right)
\end{array}
$$


we arrive at the expression

$$
\begin{aligned}
\sigma_{33}^{0}\left(x_{1}, x_{2}, x_{3}\right)=\frac{1}{2}[ & f\left(x_{1}, x_{2}, 1\right)-f\left(x_{1}, x_{2},-1\right)+\left(\int_{-1}^{x_{3}}-\int_{x_{3}}^{1}\right) g\left(x_{1}, x_{2}, \chi\right) d \chi \\
& \left.+\left(x_{3}^{3}-3 x_{3}\right) m_{\alpha \beta, \alpha \beta}+3\left(1-x_{3}^{2}\right) m_{\alpha \beta} \bar{u}_{3, \alpha \beta}-2 x_{3}\left(\bar{\sigma}_{\alpha \beta} \bar{u}_{3, \alpha}\right)_{, \beta}\right] .
\end{aligned}
$$

This used the desired boundary values: $\sigma_{33}^{0}\left(x_{1}, x_{2}, \pm 1\right)= \pm f\left(x_{1}, x_{2}, \pm 1\right)$, for substitution into the identity. However, it is not immediately evident that the given expression satisfies these conditions. By evaluating at $x_{3}=1$, we find

$$
\sigma_{33}^{0}\left(x_{1}, x_{2}, 1\right)=f\left(x_{1}, x_{2}, 1\right)-\bar{f}\left(x_{1}, x_{2}\right)-\left[m_{\alpha \beta, \alpha \beta}+\left(\bar{\sigma}_{\alpha \beta} \bar{u}_{3, \alpha}\right)_{, \beta}\right] .
$$

Now, from Eq. (26), by an application of the divergence theorem, we find that the bracketed term above is $-\bar{f}$; so $\sigma_{33}^{0}\left(x_{1}, x_{2}, 1\right)=f\left(x_{1}, x_{2}, 1\right)$. The boundary condition at $x_{3}=-1$ follows in the same way, and we have consistent constructions for all of the three-dimensional approximating functions.

Using the identities (23) we can write all these functions in terms of the displacement $\overline{\mathbf{u}}$. The following theorem summarizes our result.

TheOREM. Let $\overline{\mathbf{u}}$ be a vector field in $\mathcal{U}$ with $\bar{u}_{1}$ independent of $x_{2}$, and suppose that $\overline{\mathbf{u}}$ solves Eqs. (24) for any $v \in H^{1}(\omega)$ and (26) for any $v \in \mathcal{U}$, using the identities (23) to define $\bar{\sigma}_{\alpha \beta}$ and $m_{\alpha \beta}$. Then the following functions on $\Omega$ can be constructed:

$$
\begin{gathered}
u_{1}^{0}=\bar{u}_{1}, \quad u_{2}^{0}=\bar{u}_{2}-x_{3} \bar{u}_{3,2}, \quad u_{3}^{0}=\bar{u}_{3}, \\
\sigma_{11}^{0}=E \bar{u}_{1,1}, \quad \sigma_{12}^{0}=G\left(\bar{u}_{2,1}+\bar{u}_{3,1} \bar{u}_{3,2}-2 x_{3} \bar{u}_{3,12}\right), \\
\sigma_{22}^{0}=E\left(\bar{u}_{2,2}+\nu \bar{u}_{1,1}+\frac{1}{2}\left(\bar{u}_{3,2}\right)^{2}-x_{3} \bar{u}_{3,22}\right) .
\end{gathered}
$$

If further $\overline{\mathbf{u}}$ has the following regularity: $\bar{u}_{\alpha} \in W^{(1,4)}(\omega)$ for $\alpha=1,2 ; \bar{u}_{1,11}, \bar{u}_{2,12}, \bar{u}_{2,22}$, and $\bar{u}_{3,11} \in L^{4}(\omega)$; and $\nabla v, \nabla v_{, 2} \in L^{2}(\omega)$ for both $v=\bar{u}_{3,12}$ and $\bar{u}_{3,22}$, then the missing approximate stresses are given by

$$
\begin{gathered}
\sigma_{13}^{0}=-G\left(1-x_{3}^{2}\right) \bar{u}_{3,122}, \quad \sigma_{23}^{0}=-\left(1-x_{3}^{2}\right)\left(\frac{E}{2} \bar{u}_{3,222}+G \bar{u}_{3,112}\right), \\
\sigma_{33}^{0}=\frac{1}{2}\left\{f\left(x_{1}, x_{2}, 1\right)-f\left(x_{1}, x_{2},-1\right)+\left(\int_{-1}^{x_{3}}-\int_{x_{3}}^{1}\right) g\left(x_{1}, x_{2}, \chi\right) d \chi\right. \\
+x_{3}\left[\left(3-x_{3}^{2}\right)\left(\frac{E}{3} \bar{u}_{3,2222}+\frac{4 G}{3} \bar{u}_{3,1122}\right)\right. \\
-2\left(E\left\{\left(\bar{u}_{1,1} \bar{u}_{3,1}\right)_{, 1}+\left[\left(\bar{u}_{2,2}+\nu \bar{u}_{1,1}+\frac{1}{2}\left(\bar{u}_{3,2}\right)^{2}\right) \bar{u}_{3,2}\right]_{, 2}\right\}\right. \\
\left.\left.+G\left\{\left[\left(\bar{u}_{2,1}+\bar{u}_{3,1} \bar{u}_{3,2}\right) \bar{u}_{3,1}\right]_{, 2}+\left[\left(\bar{u}_{2,1}+\bar{u}_{3,1} \bar{u}_{3,2}\right) \bar{u}_{3,2}\right]_{, 1}\right\}\right)\right] \\
\left.-\left(1-x_{3}^{2}\right)\left(E\left(\bar{u}_{3,22}\right)^{2}+4 G\left(\bar{u}_{3,12}\right)^{2}\right)\right\} .
\end{gathered}
$$


These are approximate solutions to (18), (19), and (11) in that $\sigma^{0} \in \Sigma$ and $\mathbf{u}^{0} \in V$ satisfy the boundary data exactly, and they satisfy the variational equations up to an error of the order of $\epsilon$.

7. Construction of the degenerate von Kármán problem. We now simplify the two-dimensional problem, reducing the number of unknowns. This is a "one way"procedure in that we will lose the fact that $\bar{u}_{1}$ is independent of $x_{2}$ along the way. We introduce the notation $\sim 1=2$ and $\sim 2=1$. Our principal tool is the Airy stress function, which we use to eliminate $\bar{\sigma}$ and $\bar{u}_{\alpha}$, replacing these with a scalar function. To begin, note that, assuming $\overline{\mathbf{u}}$ is sufficiently regular, applying the divergence theorem to Eq. (24) yields

$$
\bar{\sigma}_{\alpha \beta, \beta}=0 \text { in } \omega \text { and } \quad \bar{\sigma}_{\alpha \beta} n_{\beta}=h_{\alpha} \text { on } \gamma .
$$

It is well known that, so long as the $h_{\alpha}$ satisfy Eq. (12), there exists a so-called Airy stress function, $\bar{w}\left(x_{1}, x_{2}\right)$ for which

$$
\bar{\sigma}_{\alpha \beta}=(-1)^{(\alpha+\beta)} \bar{w}_{, \sim \alpha \sim \beta}
$$

and satisfying boundary conditions:

$$
(-1)^{\alpha} \frac{d}{d s} \bar{w}_{, \alpha}=h_{\sim \alpha} \text { on } \gamma .
$$

These conditions do not specify the function uniquely, but, by distinguishing a point on $\gamma$ (making it the origin of our coordinate system), these boundary conditions and the assumption $\bar{w}(\mathbf{0})=\frac{\partial}{\partial n} \bar{w}(\mathbf{0})=0$ give Dirichlet conditions:

$$
\left.\begin{array}{rl}
\bar{w}(y)=\varphi(y):=-y_{1} \int_{\gamma(y)} h_{2} d s & +y_{2} \int_{\gamma(y)} h_{1} d s \\
& +\int_{\gamma(y)}\left(x_{1} h_{2}-x_{2} h_{1}\right) d s \\
\frac{\partial \bar{w}}{\partial \mathbf{n}}(y)=\psi(y):=-n_{1}(y) \int_{\gamma(y)} h_{2} d s+n_{2}(y) \int_{\gamma(y)} h_{1} d s
\end{array}\right\} \quad \text { for all } y \in \gamma,
$$

where $\gamma(y)$ is the contour of $\gamma$ connecting $\mathbf{0}$ to $y$ in a counterclockwise fashion. Notice that Eq. (12) must hold for $\varphi$ and $\psi$ to be well-defined. For a discussion of the existence of the Airy stress function, the reader is referred to Zeidler [18, §65.7] for a classical approach, or the discussion in $[4, \S 1.5]$, where the development is done in the greater generality of Sobolev spaces. Using $\bar{w}$ and the expressions for $m$ in terms of $\bar{u}_{3}$ in (23), we can rewrite Eq. (26) as

$$
\begin{aligned}
& \int_{\omega} \frac{E}{3} \bar{u}_{3,22} \bar{v}_{, 22}+\frac{4 G}{3} \bar{u}_{3,12} \bar{v}_{, 12} \\
& \quad+(-1)^{(\alpha+\beta)} \bar{w}_{, \sim \alpha \sim \beta} \bar{u}_{3, \alpha} \bar{v}_{, \beta} d A=\int_{\omega} \bar{f} \bar{v} d A \text { for all } \bar{v} \in U .
\end{aligned}
$$

This equation is analogous to the first of the von Kármán equations, in that it is, in variational form, a fourth-order elliptic-parabolic operator acting on the vertical deflection of the centerplane of the plate, and a bilinear form acting on the deflection and the stress function (exactly the form $[u, w]$ defined in Eq. (3)).

To eliminate the $\bar{u}_{\alpha}$, we begin by noticing that by substituting the definition (31) into Eq. (23), all the components of $D^{2} \bar{w}$ can be written in terms of $\overline{\mathbf{u}}$. Motivated by 
the second von Kármán equation (2), we seek a fourth-order, symmetric, and uniformly elliptic operator whose action on $\bar{w}$ can be described by a quadratic form acting on $\bar{u}_{3}$. Here we use that $\bar{w}_{, 22}=\bar{\sigma}_{11}^{0}=E \bar{u}_{1,1}$ is a function of $x_{1}$ only; so $\bar{w}_{, 222} \equiv 0$. It is easy to see that the identity

$$
G\left(\bar{w}_{, 1111}+\bar{w}_{, 2222}\right)+E \bar{w}_{, 1212}-\nu \bar{w}_{, 2211}=E G\left[\frac{1}{2}\left(\bar{u}_{3,2}\right)_{, 11}^{2}-\left(\bar{u}_{3,1} \bar{u}_{3,2}\right)_{, 12}\right]
$$

has the appropriate properties, provided the technical condition (15) is satisfied by the engineering constants of Hooke's Law (so that the left-hand side will be a uniformly elliptic operator). Multiplying this equation by some $v \in H_{0}^{2}(\omega)$ and integrating by parts leaves the weak formulation

$$
\int_{\omega} \frac{1}{E} \bar{w}_{, \alpha \alpha} v_{, \alpha \alpha}+\frac{1-\frac{\nu}{E}}{G} \bar{w}_{, 12} v_{, 12} d A=\int_{\omega} \bar{u}_{3,1} \bar{u}_{3,22} v_{, 1} d A \text { for all } v \in H_{0}^{2}(\omega)
$$

with boundary conditions

$$
\bar{w}=\varphi \quad \text { and } \quad \frac{\partial \bar{w}}{\partial \mathbf{n}}=\psi \quad \text { on } \gamma
$$

The equations (33), (34), and (35) are consistent for $\bar{u}_{3} \in U$, and $\bar{w} \in H^{2}(\omega)$. They are nothing more than weak formulations of the equations announced in Sec. 3, Eqs. (5) and (6). To demonstrate the equivalence we need only apply the divergence theorem in the usual way to apply all derivatives to the solutions, $\bar{u}_{3}$ and $\bar{w}$, and remove the weighting applied in the original change of variables $\left(\bar{u}_{3}=\epsilon^{-1} u, \bar{w}=\epsilon w\right.$, and $\bar{f}=\epsilon^{-1} f$ for an appropriately defined $f$ ). Recall that the $E$ and $G$ used here are smaller by a factor of $\epsilon^{3}$ than the Young's modulus and shear modulus denoted by the same letters in the summary. Thus we have completed our derivation.

When the in-plane displacement functions are reconstructed from $\bar{u}_{3}$ and $\bar{w}$, they are determined only up to a rigid motion, just as in the isotropic case; solve Eq. (23) for the $\bar{u}_{\alpha, \beta}$ in terms of $\bar{u}_{3}$ and $\bar{\sigma}_{\alpha \beta}$. Recall the assumption $\bar{u}_{1,2} \equiv 0$, even though we have no assurance that $\bar{w}_{, 222}=0$. Using (31) we have the gradients of the $\bar{u}_{\alpha}$ in terms of the solutions. The displacement in the plane of any point in the simply connected $\bar{\omega}$ (say the point $\mathbf{0}$ on $\gamma$ ), in conjunction with these gradients, completely defines the displacement $\overline{\mathbf{u}}$.

8. Conclusion. We are left with the question of existence of solutions to the problem we have derived. Based on the existence theory for the linear case, which has been examined in some detail in the works of Weinacht [16], and the author [10,11, 12], and on physical considerations, there are reasonable conditions that must be met before this boundary value problem can be assured of solutions. In the linear case, all existence results for elliptic-parabolic equations of any order rely on the appearance of a uniformly elliptic lower-order operator. For the problem at hand, the appearance of a second-order uniformly elliptic operator in Eq. (5) (the elliptic-parabolic equation) can be assured by imposing appropriate restrictions on the boundary conditions for $w$. Writing Eq. (6) as $E[w]=\left(u_{, 1} u_{, 22}\right)_{, 1}$, we can decompose $w$ into $w=w_{0}+w_{1}$ in the usual way (see [4] or [18]). We will require $w_{0}$ to solve $E\left[w_{0}\right]=0$ with the boundary conditions $(7)$, while $w_{1}$ solves the inhomogeneous equation $E\left[w_{1}\right]=\left(u_{, 1} u_{, 22}\right)_{, 1}$ with homogeneous boundary conditions. Notice that this decouples $w_{0}$ from the nonlinear system; so it is determined 
by the boundary data alone. For suitable data, we can be assured that the bilinear form $\left[u, w_{0}\right]$ will be a uniformly elliptic operator acting on $u$. It is easy to see that a necessary and sufficient condition is simply that $w_{0,11} w_{0,22}-w_{0,12}^{2} \geq c>0$ on all of $\omega$. Physically this corresponds to a requirement that the force being applied along the boundary generate tension in the interior of the plate, so as to support the load, despite the lack of stiffness. With this restriction on the boundary data in place, it may be possible to demonstrate existence of solutions to the given problem. The proof in the isotropic case, first demonstrated by Berger in [1] (for $\omega$ of unspecified shape), uses the theory of monotone operators. In particular, the von Kármán problem is restated as a fixed point problem for a pseudomonotone operator in an appropriate Hilbert space. Such an approach may be fruitful here; however, because of the degeneracy, the proper space is not obvious. This is the topic of the companion article [13]. The author expects that, once existence is demonstrated, uniqueness will follow for sufficiently small loads and large tension by the inverse function theorem. The bifurcation theory for this boundary value problem should also be analogous to that of the classical von Kármán problem, but, as [16] indicates, the spectral theory for these degenerate operators is fundamentally different from that for uniformly elliptic operators.

\section{REFERENCES}

[1] M. S. Berger, On von Kármán's equations and the buckling of a thin elastic plate, I, Communications on Pure and Applied Mathematics 20, 687-719 (1967)

[2] P. G. Ciarlet, A justification of the von Kármán equations, Archive for Rational Mechanics and Analysis 73, 349-389 (1980)

[3] P. G. Ciarlet, Plates and junctions in elastic multi-structures, Recherches en Mathématiques Appliqués, Vol. 14, Masson, Paris, 1990

[4] P. G. Ciarlet and P. Rabier, Les équations de von Kármán, Springer, Berlin and New York, 1980

[5] G. Fichera, On a unified theory of boundary value problems for elliptic-parabolic equations of the second order, in Proceedings of a Symposium on Boundary Value Problems in Differential Equations (R. E. Langer, ed.), Univ. Wisconsin Press, Madison, WI, 1960, pp. 97-120

[6] M. E. Gurtin, Topics in Finite Elasticity, CBMS-NSF Series \#35, SIAM, Philadelphia, 1981

[7] W. M. Greenlee, Degeneration of a compound plate system to a membrane-plate system: A singularly perturbed transmission problem, Ann. Mat. Appl. (4) 128, 153-167 (1981)

[8] S. G. Lekhnitskii, Theory of elasticity of an anisotropic body, Mir, Moscow, 1981

[9] J. E. Marsden and T. J. R. Hughes, Mathematical foundations of elasticity, Prentice-Hall, Englewood Cliffs, NJ, 1983

[10] R. G. Root, Boundary value problems for degenerate elliptic-parabolic fourth order equations, University of Delaware, doctoral dissertation, 1988

[11] R. G. Root, Boundary value problems for degenerate elliptic-parabolic equations of the fourth order, Transactions of the American Mathematical Society 324, No. 1 (1991)

[12] R. G. Root, Existence theory of higher order elliptic-parabolic equations with an application to elasticity, Journal of Mathematical Analysis and Its Applications 54, No. 1 (1991)

[13] R. G. Root, Boundary value problems for degenerate von Kármán equations, Quart. Appl. Math. 57, 1-17 (1999)

[14] J. J. Stoker, Nonlinear Elasticity, Gordon and Breach, New York, 1968

[15] T. von Kárman, Festigkeitsprobleme im Maschinbau, Encyl. der Math. Wissenschaften 4 (4), Leipzig, 348-352 (1907-1914) 
[16] R. J. Weinacht, Asymptotic distribution of eigenvalues for a class of degenerate elliptic operators of the fourth order, Rend. Mat. (7) 6, No. 1-2, 159-170 (1986)

[17] R. J. Weinacht, Degenerate elliptic equations and spongy elastic plates, in Methoden und Verfahren der mathematischen Physik, Problems of Applied Analysis, Vol. 33, Lang, Frankfurt am Main, 1987, pp. 59-74

[18] E. Zeidler, Nonlinear functional analysis and its applications, vol. 4, Applications to Mathematical Physics, Springer, Berlin and New York, 1988 